\title{
Planeación estratégica en la Pyme de Bogotá Orientación al futuro y evasión de la incertidumbre
}

Alejandro Castaño

Ramírez

Institución Universitaria Politécnico Grancolombiano acastano@poligran.edu.co

\section{Resumen}

El futuro genera sentimientos y establece patrones de comportamiento organizacional que son realizados por las pequeñas y medianas empresas; estos comportamientos ya han sido investigados en lugares como Michigan o Australia. El propósito de este artículo es aportar en el conocimiento del tema en un contexto específico: la localidad de Chapinero en la ciudad de Bogotá, Colombia. La investigación analiza cualitativamente cómo se realizan los procesos de planeación estratégica; para ello, se efectúan mediciones culturales cuantitativas que, teóricamente, pueden relacionarse con la planeación estratégica. Los resultados nos muestran que el comportamiento organizacional de estas empresas utiliza marcos espacio temporales a corto plazo, formas emocionales de tomar decisiones, desconocimiento de herramientas de mercadeo, además de altos niveles de incertidumbre, los que motivan la adopción de herramientas de gestión y administración para realizar procesos de planeación estratégica, aun cuando no se tenga una comprensión completa de lo que significa.

Palabras clave: planeación estratégica, incertidumbre, orientación en el tiempo Pyme.

Clasificación JEL: M31 


\title{
Strategic planning in small and medium enterprises in Bogotá, Colombia Future-oriented approach and uncertainty prevention
}

\begin{abstract}
The future breeds emotions and sets up behavior patterns performed by small and medium enterprises. There has been research on those behaviors in places such as Michigan and Australia, and this paper aims to add to the knowledge on the topic in a specific context: Chapinero district in Bogotá city, Colombia. The research analyses from a qualitative approach the way entrepreneurs make strategic plans; for that purpose, we use quantitative cultural measures theoretically related to strategic planning. Results show organizational behavior patterns such as the use of short time orientations, emotional biases, ignorance of marketing tools, plus high uncertainty levels which foster the adoption of strategic planning procedures without fully understanding their meaning.
\end{abstract}

Keywords: strategic planning, uncertainty, SMEs.

JEL classification: M31

\section{Introducción}

La forma como se piensa y enfrenta el futuro comercial de una Pyme (Pequeña y Mediana Empresa) está anclada a principios y valores culturales fuertemente arraigados y que, socialmente, construyen la cultura de las personas y de las organizaciones. El futuro genera la emoción de la ansiedad a través de la incertidumbre en los seres humanos y sus comunidades; en consecuencia, cada cultura organiza maneras específicas para manejar la ansiedad y la incertidumbre. La actividad de planeación estratégica busca controlar la incertidumbre y generar escenarios prospectivos para la Pyme, pero este concepto, que viene de los referentes conceptuales de la administración, es entendido y utilizado por los empresarios de acuerdo con sus propias condiciones culturales al hacer negocios. Por lo tanto, es necesario conocer las perspectivas que estos empresarios tienen hacia el futuro y cómo se adaptan a él para favorecer su desarrollo.

En el presente artículo presentaremos los resultados de una investigación empírica con un enfoque cualitativo y cuantitativo. En la primera fase, a través de metodologías cualitativas de entrevista y etnografía, se exploró la manera como 
se desarrolló el proceso de planeación estratégica en tres empresas; en la segunda fase, a través del cuestionario VSM (Value Survey Model) se plantean variables culturales que pueden aportar a la investigación sobre planeación estratégica de manera cuantitativa.

\section{Cultura y la planeación estratégica}

Las estrategias comerciales están atadas a la cultura; aquellas que logran adaptarse a paradigmas culturales son consideradas exitosas. Un ejemplo específico es la estrategia comercial denominada Gianxi, en China, la cual determinó la entrada de grandes marcas multinacionales al mercado chino como resultado del control constante del gobierno sobre las actividades comerciales. Este filtro político devino en la búsqueda del Gianxi o en la posibilidad de tener relaciones sociales con personas influyentes del gobierno con el fin de hacer viables los proyectos de alianzas comerciales entre las multinacionales y las nacientes empresas chinas (Liu y Roos, 2006: 433). Este ejemplo nos muestra cómo la cultura crea las condiciones comerciales en las que se desarrollan las empresas y condiciona sus estrategias.

En cada empresa, esta realidad cultural se muestra en la manera en que se toman decisiones, al mismo tiempo que dichas decisiones estratégicas hacia el futuro son el resultado de una adaptación cultural de los empresarios en un medio ambiente comercial. Así, obtenemos una imagen de una cultura gracias a la forma de hacer negocios en un determinado contexto.

El primer elemento conceptual muestra un patrón de comportamiento de la Pyme frente al futuro: la planeación estratégica, la cual definiremos desde un punto de vista cultural y es la que define la investigadora Eileen Mulhare (1999: 323): "una variedad de prácticas administrativas por las que la compañía trata de alinear las acciones con las metas".

Los fenómenos de la planeación estratégica han sido abordados ampliamente por Henry Mintzberg, para quien los empresarios de la Pyme desarrollan negocios a partir de modelos de emprendimiento, donde la planeación estratégica comercial tiene un alto componente informal, es manejada fluidamente por el emprendedor, con flexibilidad y adaptabilidad a los cambios (Mintzberg, 1981, p. 323). Solamente cuando una empresa ha crecido por fuera del alcance de control de su emprendedor se ve obligada a hacer un proceso de planeación formal (Mintzberg y Waters, 1982, p. 491; Hill, Nancarrow y Right, 2002, p. 362; Masurel y Smit 2000, p. 96). 
Actualmente, se afirma que la planeación estratégica adopta patrones de comportamiento de acuerdo con el ciclo de desarrollo de la empresa: va de un ciclo de emprendedor — informal— a uno de planeación — formal—, lo que afecta directamente la identidad de la empresa.

En la literatura académica, esta conceptualización hecha por Mintzberg se encuentra con frecuencia, aun cuando esté expresada con otros términos. Gibbons y O'Connor utilizan estas diferencias al ponerlas en términos de formas de pensamiento relacionadas con la planeación: el formalismo sinóptico y las formas adaptativas de aprendizaje (2005, p. 171), abordando el tema desde una perspectiva cognitiva. Otros autores hacen alusión a este concepto utilizando un lenguaje diferente: lo explícito (formal-modo de planeación) y lo implícito (formas adaptativas de aprendizaje-modo emprendedor), desde un punto de vista comunicativo (Hill, Nancarrow y Right 2002, p. 367).

Un segundo elemento conceptual con relación a la planeación estratégica está en el alto componente emocional e intuitivo que determina cómo se toman las decisiones acerca del futuro. Esto contrasta con los patrones de planeación estratégica en empresas grandes, donde las formas complejas de organización y la amplia especialización del trabajo devienen en una forma "racional" de asumir los procesos de planeación estratégica (Cassar y Gibson, 2007, p. 284; Culkin y Smith 2000, p. 148; Jocumsen, 2004, p. 662).

En la literatura, el patrón de comportamiento emocional e intuitivo se vincula con la personalidad del emprendedor, la cual lo lleva a una planeación intuitiva que es altamente optimista, extrema y basada en información llamativa, pero no necesariamente adecuada (Cassar y Gibson, 2007, p. 301). Más aún, para algunos autores existe una "simbiosis" de los emprendedores con sus empresas, encontrando trayectorias estratégicas que mezclan territorios de sus vidas personales con los del negocio (Culkin y Smith, 2000, p. 154; Jocumsen, 2004, p. 669). Aborda el tema identificando tendencias en las personalidades de los emprendedores y, por ejemplo, establece la importancia de elementos psicológicos como el locus de control con relación a la toma de decisiones estratégicas (McDonalds, Spear y Parker, 2004, p. 42; Rauch, Frese y Sonnentag, 2000, p. 35).

La caracterización de dichos elementos ha llevado a otros académicos a explicar, a través de modelos, las actitudes organizacionales y los emprendedores. Uno de estos modelos de personalidad es el de Miles y Snow (1978, p. 54), quienes esta- 
blecen tipologías estructurales dicotómicas: prospectivos-defensivos o analistasreactivos. Otro modelo es el de Culkin y Smith, donde se tipifica a la indiscutible cabeza de hogar; los casados y sufriendo; el esposo hágalo usted mismo; y los compañeros ilustrados, quienes, por ejemplo, tienen una posición progresista y enfocada hacia el mundo comercial (2000, p. 152).

Un tercer elemento conceptual alrededor de la planeación estratégica es que el emprendedor, en este tipo de empresas, se ve en posiciones no especializadas y toma decisiones sobre diversos temas que involucran a la empresa: finanzas, insumos, ventas, mercadeo, recursos humanos, logística (Culkin y Smith, 2000, p. 156).

Un cuarto y último elemento conceptual por considerar alrededor del futuro es la influencia de la red social del emprendedor: está rodeado de relaciones sociales, las cuales están atravesadas por estructuras familiares que generan espacios de acompañamiento y apoyo para la toma de decisiones estratégicas. Este cuarto elemento es abordado por la literatura en temas como la relación entre las reuniones familiares sobre negocios y la planeación estratégica, lo que resulta no concluyente; es decir, una red social solidaria y afianzada alrededor del emprendedor no necesariamente genera procesos de planeación estratégica para enfrentar el futuro (Tower, Gudmundson, Schierstedt y Hartman, 2007, p. 91).

En resumen, los elementos conceptuales previamente descritos a partir de la literatura existente señalan que la Pyme teóricamente presenta los siguientes comportamientos de la planeación estratégica y el futuro: a) procesos informales que las hace bastante flexibles, b) un carácter intuitivo, c) no especializado-multifuncional, d) altamente emocional, e) dependiente de la personalidad de cada emprendedor, quien está generalmente inmerso y f) el emprendedor es influido por una red de apoyo familiar.

\section{Cultura Pyme en Colombia}

El anterior panorama debe ser contextualizado; por lo tanto, a continuación se presenta un breve análisis de estas condiciones dentro del contexto cultural comercial en Colombia, a partir de la literatura académica disponible.

De acuerdo con la investigación realizada por Ana Calle y Víctor Tamayo en Antioquia, la Pyme tiene las siguientes características: se dedican a prestar servicios (49.9\%), al comercio $(21.5 \%)$ y a la industria (19.3\%); tienen altos niveles de 
informalidad, expresada en que no pagan impuestos (53.5\%), no llevan registros contables (42\%) y no tienen registro mercantil (45\%); tienen baja capacidad para realizar actividades rentables y con potencial de expansión; tienen bajos niveles de asociatividad; hay estrechez de los mercados a los que dirigen sus productos, los cuales son mayoritariamente mercados locales, con consumidores de bajos ingresos, con bajos requisitos de calidad; tienen un bajo nivel tecnológico (utilización de maquinarias y equipos desactualizados) y de capacitación del recurso humano; tienen un limitado acceso al sector financiero, pues obtienen la financiación de los ahorros acumulados por la familia y de los ingresos de trabajos anteriores (72\%) (Calle y Tamayo, 2005, p. 155).

Estas características hablan del contexto social y económico donde se mueve la Pyme, reforzando los elementos conceptuales identificados en la literatura internacional al encontrar empresas con alta informalidad donde los procesos no son formalizados, la planeación se hace con carácter intuitivo con un alto nivel de emocionalidad y con una interacción familiar fuerte implícita en la financiación familiar de los negocios.

La investigación del profesor de la Universidad Nacional de Colombia, sede Manizales, Gregorio Calderón y su semillero de investigación sobre Pyme en el eje cafetero permite deducir una estructura organizacional Pyme que se articula a través de "una organización poco burocratizada en su estructura, con un número reducido de niveles jerárquicos y con una alta informalidad en el manejo de las relaciones" (Calderón, 2006, p. 69). Esto ha devenido en características organizacionales flexibles y en patrones laborales no especializados multifuncionales alrededor de fenómenos como "la polivalencia y la adaptación rol-individuo" (Calderón, 2006, p. 69).

La alta preponderancia de la figura del emprendedor en la Pyme ha generado un patrón que afecta directamente la toma de decisiones y marca una tendencia de simbiosis entre la personalidad del emprendedor y la forma en que se establece la planeación estratégica. Esto se observa en un patrón de comportamiento que resalta Calderón, a saber: no obstante que la Pyme genera espacios de participación para sus empleados, esta participación es restringida al compartir ideas y perspectivas sin que afecten o influyan las decisiones estratégicas, las cuales residen en el emprendedor y su círculo íntimo (Calderón, 2006, p. 69).

En Colombia, la volatilidad del ambiente comercial y social no ha generado una actitud proclive al cambio y la adaptación al mismo en la Pyme; como señala 
Calderón, los cambios a los que se ve obligada la Pyme son una respuesta a las necesidades del mercado, no una estrategia para ser competitivo en él, de acuerdo con las investigaciones hechas previamente (Calderón, 2006, p. 67).

Por lo tanto, la planeación estratégica entendida desde un punto de vista tradicional no parece tener lugar en la Pyme en Colombia, ni como previsión del futuro ni como proceso formal, puesto que reportan las investigaciones que la Pyme no está acostumbrada a tener visiones de largo plazo, aun cuando su esquema organizacional genere altos vínculos emocionales con sus dueños y empleados, debido a la cercanía y dependencia mutua. Hay, por el contrario, una visión cortoplacista (Calderón 2006, p. 69; Calle y Tamayo, 2005, p. 156) que inhibe el pensamiento estratégico y las perspectivas de futuro lejano.

Hasta este punto hemos esbozado algunos de los elementos que investigaciones empíricas han abordado sobre la planeación estratégica en la Pyme.

En el nivel internacional podemos sintetizar que las características de la planeación estratégica son procesos informales; carácter intuitivo; multifuncionalidad del emprendedor y planeador; carácter altamente emocional; dependiente de la personalidad de emprendedor; y vinculado a redes familiares.

En el nivel nacional encontramos que las mismas características de la planeación estratégica son resaltadas: procesos informales; polivalencia y multifuncionalidad del emprendedor y planeador; carácter altamente emocional; preponderancia de la figura del emprendedor; y vinculado a redes familiares.

Con relación al contexto comercial donde se desenvuelve la Pyme, en Colombia encontramos las siguientes características: alta informalidad; baja rentabilidad; baja asociatividad; operación en mercados locales; bajo nivel de uso de tecnología; y poca financiación y tendencia de autofinanciación.

\section{Planeación estratégica y contexto cultural}

Los paradigmas de administración actuales consideran la realización de procesos de planeación, los cuales han sido señalados por tradiciones académicas comerciales y administrativas, como requisito indispensable para el buen desempeño empresarial (Mulhare, 1999, p. 324). 
Sin embargo, otras perspectivas académicas muestran que la planeación estratégica no es una condición inherente del éxito y por el contrario dificultan la flexibilidad, la innovación y la creatividad en las empresas (Jordan, 2003, p. 46). En la literatura se puntualiza que solamente en culturas donde hay una alta tendencia a la evasión de la incertidumbre, como es el caso de la cultura alemana, la planeación estratégica es exitosa. Esto hace contraste con otras culturas, como la irlandesa, donde la incertidumbre se maneja con una alta dosis de flexibilidad y, en consecuencia, la planeación se percibe como un proceso costoso, que demanda tiempo y cuyo resultado es fácilmente abandonado, dependiendo de las necesidades que impone el mercado a la Pyme (Rauch, Frese y Sabine Sonnentag, 2000, p. 37).

El trabajo de Geert Hofstede y Geert Jan Hofstede (2005, p. 86) aborda el manejo de la incertidumbre como una dimensión cultural. Esta dimensión corresponde a la tendencia hacia la evasión de situaciones inciertas, cambios constantes y caos, en los miembros de una determinada cultura, que son situaciones relacionadas con la emoción de la ansiedad y que llevan, según los autores, hacia actitudes proclives a la planeación estratégica.

Estos cuestionamientos a la funcionalidad universal de la planeación estratégica resaltan las diferencias en los valores culturales entre las comunidades como primordiales para dicha planeación.

En este orden de ideas, en la presente investigación se parte de una exploración cualitativa de la actividad de planeación estratégica, identificando la naturaleza de su procedimiento y contexto, además de explorar las variables que las investigaciones sobre cultura empresarial de Geert y Geert Jan Hofstede asocian con la planeación estratégica: la evasión de la incertidumbre y la orientación a largo plazo para luego explorar estas variables de manera cuantitativa y validar herramientas metodológicas que permitan estudiar el fenómeno de la planeación estratégica en la Pyme desde una perspectiva cultural.

Cabe anotar en este momento que la importancia de la cultura en fenómenos económicos no ha sido solamente señalada por la antropología, sino que los mismos economistas han percibido esta importancia y, por ejemplo, Michael Porter indica que la cultura de los países tiene influencia en su desempeño económico (1990, p. 36). En consonancia, otras investigaciones económicas y administrativas han contemplado problemas económicos desde una perspectiva cultural (Hofstede, 1980, p. 35; Franke, Hofstede y Bond, 1991, p. 166; Trompenaars y Hampden-Turner, 
1998, p. 16), las cuales concluyen que las diferencias en los valores culturales, más aún que las diferencias materiales y estructurales, son las que conducen a un país hacia un buen desempeño económico (Franke, Hofstede y Bond, 1991, p. 166).

\section{Fase cualitativa}

La presente investigación utilizó metodologías de entrevista y etnografía como metodologías de investigación cualitativa con el fin de explorar las actividades de planeación estratégica y delimitar la presencia de los elementos conceptuales reseñados en la literatura internacional y de Colombia.

Los casos fueron explorados a través de entrevistas cualitativas en profundidad (Denzin y Lincoln, 2005, p. 135), de las cuales se aplicaron cinco en cada caso. Estas entrevistas se complementaron con seguimientos etnográficos, los cuales fueron participaciones en eventos de planeación estratégica de las empresas; se participó en un total de 18 eventos (seis por empresa). Este trabajo de campo se realizó durante los meses de enero, febrero y marzo de 2009.

La entrevista cualitativa es definida por los especialistas en metodología como "un intercambio de ideas, significados y sentimientos sobre el mundo y los eventos" (Bonilla-Castro y Rodríguez, 1997, p. 75). Se optó por un formato de entrevista cualitativa denominado entrevista narrativa, donde "las preguntas se formulan en torno a un asunto que se explora ampliamente, sin usar ninguna guía que delimite el proceso" (Bonilla-Castro y Rodríguez, 1997, p. 76). A través de estas entrevistas se reconstruyó la historia empresarial y, a lo largo de ésta, se identificaron los procesos de formación de planeación estratégica.

De las empresas participantes en la fase cualitativa se han escogido tres casos que muestran composiciones organizacionales y actitudes diferentes con relación a la planeación estratégica: el primero, el de una pareja que se independiza y consolida su empresa: Productos Alimenticios el Pilón; el segundo, el de una empresa familiar con un negocio ya consolidado: Madechem Ltda.; y el tercero, el de una empresa no familiar, con varios años de trabajo, pero con una intención de crecimiento: Grupo ACLAB Ltda. 


\section{Presentación de casos}

La información cualitativa la analizaremos con relación a los elementos conceptuales identificados en la literatura internacional y nacional, en torno a los patrones de comportamiento y en torno a la planeación estratégica identificados previamente.

\section{Características de la planeación estratégica de Productos Alimenticios El Pilón}

Procesos informales: los emprendedores realizan un ejercicio de planeación estratégica cada año, en el cual reúnen información interna y de comportamiento de los mercados para plantearse objetivos y metas para cada año. Este procedimiento queda consignado en un documento final. La forma en que se desarrolla esta planeación es bastante amplia, permitiéndoles abandonar lo planeado frente a nuevas circunstancias.

Carácter intuitivo: el carácter intuitivo de la planeación estratégica se evidencia en el momento en que Productos Alimenticios El Pilón tuvo acceso al hipermercado Carrefour, de continuidad con una estrategia de entrada a supermercados. Pero el crecimiento alcanzado hasta el momento fue interrumpido, puesto que, en un comienzo, con estrategia agresiva de competencia en el punto de venta, es decir, con más impulsadoras y degustaciones y promociones, las ventas subieron. Eso los llevó a generar mayores niveles de producción. Pero en varios meses el hipermercado les hizo amplias devoluciones. La emprendedora puso tanto mercaderistas como impulsadoras en cada hipermercado para garantizar que su producto fuera exhibido y tuviera rotación. Pero la situación no cambió, las ventas bajaron y por estar dedicados al hipermercado descuidaron los mercados que ya tenían colonizados. Varios meses después toman la decisión de salir del hipermercado, manifiesta que de no hacerlo hubiera significado la bancarrota de la empresa.

Esta situación de escoger una estrategia en Carrefour denota una alta emocionalidad en la planeación estratégica, que decide asumir una forma de distribución sin explorar de manera juiciosa las condiciones que implica esta nueva forma de distribución.

Vinculado a redes familiares: la planeación estratégica en esta empresa está dirigida por la pareja de emprendedores, quienes realizan este proceso de la manera descrita en procesos informales. 
En esta empresa no se observó una tendencia de la emprendedora a ser multifuncional, ya que la unidad familiar asume otras funciones como la financiera dentro de la empresa.

La dependencia de la personalidad del emprendedor se observa en esta empresa en la medida en que la formación de los emprendedores, quienes han estado relacionados con empresas multinacionales laboralmente y han adoptado prácticas administrativas propias de este tipo de empresas.

Características de la planeación estratégica de Madechem Ltda.

Procesos informales: la empresa Madechem es resultado del emprendimiento de un hombre, quien trabajó durante muchos años como visitador médico. Hace 25 años el emprendedor decidió formar su empresa y se puso a la cabeza de una empresa de distribución de principios farmacéuticos activos provenientes de una multinacional llamada Chemo. Como visitador, el emprendedor consolidó relaciones estrechas con la industria farmacéutica en Bogotá, las cuales, aunadas a los eventos de mercadeo que hace Chemo, generó la estrategia con la que el emprendedor ha competido durante los años de vida de su empresa: una estrategia fuertemente anclada a la asistencia técnica y científica, alrededor de lazos sociales de amistad y camaradería. Esta estrategia ha sido exitosa y no ha sido necesario cambiarla para adaptarse al mercado, lo que demuestra que la planeación estratégica depende en gran medida de la personalidad del emprendedor.

En consecuencia no reporta realizar una planeación estratégica formalizada. Antes bien, las estrategias de mercadeo están basadas en las directrices y desarrollos de la multinacional, lo que denota una no multifuncionalidad del emprendedor.

Vinculado a redes familiares: Madechem ha seguido el esquema de crecimiento familiar y ahora toda la familia está involucrada con el negocio de una u otra manera. El presidente es el emprendedor, su hijo es el segundo al mando y también, como el emprendedor, maneja los clientes. Las decisiones son tomadas por ellos dos, claro, en armonía con las directrices de la multinacional Chemo.

No se observaron características propias de un carácter intuitivo, en la medida en que no se realiza una planeación estratégica, así como tampoco se observó un carácter altamente emocional en virtud de la misma razón. 
Las estrategias y la planeación de ACLAB Ltda.

El grupo ACLAB (Aseguramiento de Calidad en Laboratorios) está conformado por cinco mujeres profesionales que se han asociado. Todas trabajaron en algún momento en alguna empresa colombiana de producción o distribución de alimentos (Alpina, Carulla, Instituto Nacional de Salud, INVIMA). Esas experiencias las llevaron a desarrollarse como capacitadoras en calidad y asociarse como ACLAB.

Procesos informales: los procesos de planeación estratégica de esta compañía fueron claramente identificables al ser procesos completamente formalizados, pues sostiene reuniones de planeación estratégica, las cuales implican altos niveles de verbalización, generando instrumentos escritos de control sobre las actividades no solamente de planeación, sino sobre la conformación de la empresa como tal. Se pudo observar que el insumo o herramienta principal para la realización de estrategias es la matriz DOFA.

Carácter no especializado-multifuncional: el proceso de planeación surgió y fue liderado por una de las socias de la Pyme, quien no solamente ha recibido instrucción en administración (tiene un MBA), sino que es responsable de la adecuada conceptualización del proceso de planeación estratégica en el grupo, lo que se ve reflejado en que en un momento de las sesiones de planeación observadas ella conecta su computador al video-beam y muestra a las otras socias una presentación muy breve y concisa sobre la ventaja competitiva, a la manera de Michael Porter.

La disposición espacial de trabajo del grupo es circular, garantizando la participación de todos los socios y generando un sentido de igualdad y solidaridad alrededor de la construcción de los procesos.

Carácter intuitivo: las socias de ACLAB tienen, según ellas mismas, un enfoque técnico y poco empresarial, lo que las lleva a una planeación altamente intuitiva. Esto es visible cuando el grupo debe delimitar estrategias de competencia. En ese momento las socias dejan de participar activamente y esperan la guía de la líder para realizar el ejercicio, lo que denota una especialización de las funciones en la Pyme y la no multifuncionalidad. La manera de abordar este ejercicio parte de la utilización de la matriz DOFA, a través de la cual concretan una perspectiva sobre el mercado que puede tener la empresa, que son básicamente los laboratorios de alimentos. Identifican otros profesionales dedicados a la capacitación y la principal competencia son los programas de aseguramiento de la calidad liderados por el 
gobierno. Por lo tanto, enfocan su estrategia comercial hacia la industria privada, considerando algunos proyectos gubernamentales que ellas pueden llegar a administrar como ACLAB.

Una de las situaciones en las que las socias hacen mayor énfasis es en su propio desconocimiento del mercado, lo que denota lo dependiente de la personalidad de las emprendedoras con relación a la planeación estratégica. Esto les genera ansiedad y las obliga a pensar en mecanismos para identificar con mayor precisión las tarifas y productos que se están ofreciendo en el mercado.

\section{Comparación de resultados fase cualitativa}

Una de las primeras diferencias que surgen al comparar los tres casos es la utilización (o no) de procesos de planeación estratégica. El caso en que no se realiza planeación estratégica nos muestra una forma tradicional de las empresas en Bogotá: aquellas que están inmersas en una red social que las soporta y les brinda estabilidad. La estrategia es implícita, basada en el fortalecimiento de la red social del empresario y su familia, y no necesita ser documentada, formalizada, ni formulada a través de procesos administrativos. El futuro en estas condiciones está atado a la pertenencia a dicha red y debido a que la pertenencia se ha construido durante mucho tiempo no hay ansiedad con respecto al futuro. En este caso podemos ver la alta preponderancia de la figura y personalidad del emprendedor para la empresa, pues de él provienen las relaciones comerciales y su mantenimiento.

Los otros dos casos muestran patrones de comportamiento diferentes, con los que las empresas enfrentan nuevas situaciones de mercado, de ansiedad e incertidumbre, donde se observa la formalización de procesos de planeación estratégica. Esto muestra características como la utilización de un modelo de planeación estratégica de emprendimiento, con alta informalidad (aunque se elaboren documentos y registros), flexibilidad y adaptabilidad.

En los casos que llevan a cabo procesos de planeación estratégica se puede observar la presencia de un alto componente emocional e intuitivo, evidente en la determinación de estrategias sin información, que obedecen a imaginarios culturales. El caso de Productos Alimenticios El Pilón con relación a la entrada en hipermercados muestra este patrón de comportamiento. En el caso de ACLAB, ellas explican este patrón de comportamiento como resultado de su poca formación comercial. 
La presencia de la red familiar también fue constante en dos de los tres casos explorados. Sin embargo, la composición organizacional de las Pyme es variada y nuestro tercer escogido muestra una empresa no-familiar, que es el resultado de una amistad que surge en la universidad. En empresas de este tipo las cargas laborales están más repartidas y no se observa que uno de los socios tenga más poder que los otros, aun cuando alguien lidere los procesos administrativos por su formación educativa. La comparación nos deja ver que las empresas familiares, como Madechem y Productos Alimenticios El Pilón, tienen entonces una estructura de poder más vertical, donde la concentración de las decisiones sobre el futuro de la Pyme está en manos de los miembros de la familia con poder de decisión. En el tercer caso, ACLAB, los espacios se abren a los socios a través de reuniones de planeación estratégica.

Esto va de acuerdo con los resultados de otras investigaciones académicas hechas en Colombia y previamente mencionadas, donde se identifica la concentración de poder de decisión en unos cuantos miembros de la familia.

Los horizontes de tiempo en las dos empresas que hacen planeación fueron a un año, lo que nos muestra una tendencia al corto plazo, el cual es necesario corroborar en la población a través de mecanismos cuantitativos.

\section{Fase cuantitativa}

La metodología del Value Survey Model (VSM) fue desarrollada por el profesor Geert Hofstede, ahora profesor de la Universidad de Maastrich en Holanda, y fue el instrumento (en sus versiones originales) utilizado en la investigación de valores organizacionales que realizó el profesor Hofstede en la década de los setenta en compañías pertenecientes a IBM en 18 países del mundo.

Con la publicación de los resultados de la investigación, vistos desde una perspectiva cros-cultural y de tendencia nacional, surge la encuesta VSM en 1980, que queda consignada en el libro Culture's Consequences (Hofstede, 1980, p. 125), uno de los más citados en el Social Science Citation Index de acuerdo con Tony Fang (2003, p. 347).

El índice de la evasión de la incertidumbre está relacionado con la tendencia a manejar el riesgo y la emoción subyacente: la ansiedad, a través de la valoración y realización de la planeación del trabajo y por ende la planeación estratégica (Hofstede, 2005, p. 90). 
Además, incluimos la medición del Índice de Orientación a Largo Plazo para contextualizar cuantitativamente la disposición del emprendedor a pensar de esta manera y profundizar sobre este elemento distintivo de la Pyme en Colombia, previamente identificado en la literatura académica (Calle y Tamayo, 2005, p. 165; López y Calderón, 2006, p. 29; Calderón, 2006, p. 69).

La encuesta VSM es un cuestionario estructurado de 26 preguntas: 18 con escala likert, 2 de opción múltiple y 6 demográficas. El formato más reciente a la fecha de realización del trabajo de campo es del año 2008, pero una versión en inglés; por ello, utilizamos la versión oficial en español del año 2004 (ver anexo 1). Es de uso libre para fines académicos, como es el caso de esta investigación.

Al formato le fueron añadidas preguntas de identificación de la Pyme como el nombre, la dirección, el contacto por internet y el teléfono, con el fin de realizar el control de calidad; además, incluimos una pregunta dicotómica sobre la realización de planeación estratégica, que si bien puede recolectar datos "aspiracionales", puede también ser un buen indicio de la realidad de la planeación estratégica en Pyme.

La muestra corresponde a la población de Pyme en la localidad de Chapinero, en las cuales se encuestó al emprendedor, dueño o gerente. Para calcular el tamaño de la muestra se definió a partir de la existencia de un total de 20,778 unidades productivas en la localidad; el 6\% de estas son Pyme, así que el universo corresponde a: 1,247 unidades productivas Pyme (Cámara de Comercio de Bogotá y Universidad Santo Tomás, 2008, p. 7).

Por lo tanto, con un 95\% de nivel confianza y un 5\% de margen de error, calculamos una muestra total de 294 Pyme, de las cuales el 84\% (247) deben ser pequeñas y el $16 \%$ (47) deben ser medianas.

Una Pyme se define para la investigación como una unidad productiva que tiene una característica doble. La pequeña empresa tiene entre 11 y 200 empleados, mientras que la mediana empresa tiene entre 51 y 200, de acuerdo con la ley 905 de 2004 (Congreso de Colombia, 2004, p. 1).

La encuesta se aplicó de manera asistida, buscando la interacción personal con los encuestados. La información se tabuló en Excel y se procesó de acuerdo con las fórmulas de los índices del VSM. Asimismo, esta información se procesó para 
calcular los índices de la Evasión de la incertidumbre y Orientación al largo plazo, que se calculan con las siguientes fórmulas:

Índice Evasión Incertidumbre $=+25 \mathrm{~m}(13)+20 \mathrm{~m}(16)-50 \mathrm{~m}(18)-15 \mathrm{~m}(19)+120$. En esta fórmula m(13) es el promedio de la pregunta 13, y así respectivamente.

Índice de Orientación al largo plazo = $-20 \mathrm{~m}(10)+20 \mathrm{~m}(12)+40$.

\section{Resultados fase cuantitativa}

Se aplicó la encuesta a un total de 329 Pyme (el listado completo se puede consultar en el Anexo 2), superando la muestra delimitada previamente (294). Participaron: 280 pequeñas empresas y 49 medianas empresas. Esta encuesta se aplicó durante el mes de agosto del año 2009 por 80 estudiantes del curso de Investigación Aplicada al Mercadeo del programa académico de Mercadeo y Publicidad del Politécnico Grancolombiano. Los participantes fueron obtenidos a través de las redes de los estudiantes, es decir, referidos.

El índice de evasión de la incertidumbre, uno de los objetivos de medición, marcó un total de 73, lo que denota una tendencia alta a la evasión de la incertidumbre y el control de la ansiedad entre los empresarios. No se encontraron diferencias marcadas entre pequeñas y grandes empresas ( 72 medianas y 74 pequeñas). Esto nos ayuda a comprender por qué en la mayoría de los casos en los que se hizo seguimiento cualitativo (2/3) encontramos presencia de actividades de planeación estratégica. A nivel cuantitativo, el 74\% (274/329) de la muestra manifiesta realizar planeación estratégica, resultado que va acorde con los patrones culturales descritos previamente a nivel cualitativo, donde $2 / 3$ casos hacen planeación estratégica.

El índice de orientación al largo plazo, nuestro segundo objetivo de medición, es relativamente bajo: 54, lo que indica que nuestros empresarios no tienen una orientación marcada al largo plazo, situación que la literatura académica ha identificado. Esto explica la elaboración de planes estratégicos a corto plazo (2/3 de los participantes de la muestra cualitativa los hacen a un año). Tampoco se encontraron diferencias entre ambos tipos de empresas. 


\section{Conclusión}

El futuro para la Pyme es asumido por los emprendedores a través de la realización de actividades de planeación estratégica con características altamente informales, donde se formaliza, en un documento escrito, la planeación estratégica, pero también donde, dicha planeación, es flexible y susceptible de abandonarse; se caracteriza también por una tendencia intuitiva, altamente emocional y toma de decisiones desde posiciones multifuncionales del emprendedor, donde la planeación estratégica depende de su personalidad y de las redes familiares que le ayudan a pensar el futuro.

La medición cuantitativa nos permite deducir que hay altos niveles de ansiedad en la forma de incertidumbre, lo que explica el uso de la planeación estratégica bajo un esquema flexible, adaptable e informal, dentro de un escenario cortoplacista, lo que muestra una tendencia cultural diferente a la que se mostró en otras latitudes; además de una utilización de este tipo de herramientas de administración, aun cuando no son del todo operativas. Esto indica una cultura con alta incertidumbre, donde la planeación estratégica es altamente valorada, aunque su utilización se haga de manera particular.

Los patrones culturales Pyme están altamente influidos por la dinámica familiar, con esquemas de poder verticales y figuras centrales multifuncionales que concentran las decisiones hacia el futuro.

El futuro para la Pyme, por lo tanto, es incierto; no se sabe cómo enfrentarlo claramente, como muestra la medición cuantitativa del alto Índice de Evasión de la Incertidumbre. Esto se debe, por un lado, a la falta de formación comercial y administrativa de los emprendedores, además de la falta de información, y, por otro, por la ambigüedad y el caos del medio ambiente comercial.

La tendencia emocional e intuitiva de la planeación estratégica, aunada a su carácter cortoplacista encontrado a través de ambas fases metodológicas pone a la Pyme en grave riesgo de supervivencia y las redes familiares de apoyo, aunque si bien sustentan al empresario financieramente, no conducen a una identidad organizacional proyectada al éxito. 


\section{Referencias}

Bonilla-Castro, E. y P. Rodríguez (1997). Más allá del dilema de los métodos. $3^{\mathrm{a}}$. ed., Bogotá: Grupo Editorial Norma-Universidad de los Andes,.

Cámara de Comercio de Bogotá, Universidad Santo Tomás (2009). Censo Empresarial de Chapinero. Extraído el 29 de julio 2009: http://camara.ccb.org. co/documentos/3399_Presentaci\%C3\%B3n_Censo_Empresarial_en_Chapinero_Web.pdf

Calle, A. M. y V. M. Tamayo (2006). Estrategia e internacionalización en las PYMES: Caso Antioquía. Cuadernos de administración, 18 (30), 139-164.

Cassar, G. y B. Gibson (2007). Forecast rationality in small firms. Journal of Small Business Management, 45(3): 283-302.

Culkin, N. y D. Smith (2000). An emotional business. A guide to understanding the motivations of small business decision takers. Qualitative market research 3 (3): 145-161.

Calderón, G. (2006). Competencias distintivas en las PYMES: un aporte desde gestión humana. Innovar 16 (27): 57-71.

Congreso de Colombia. (2004). Ley 905. En: Ley 905 de 2004. Extraído el 15 de marzo 2009: http://appserver.lasalle.edu.co/cedef/media/Ley_905_2004_ MIPYMES.pdf

Denzin, N. e Y. Lincoln (2005). Handbook of qualitative research. Urbana: Sage Publications.

Hill, J., C. Nancarrow y L.T. Right (2002). Lifecycles and crisis points in SMEs: a case approach. Marketing intelligence and planning 20 (6): 361-369.

Hofstede, G. (1980). Culture's consequences: international differences in workrelated values. Beverly: Sage publications.

Hofstede, G. y G.J. Hosftede (2005). Cultures and organizations. Nueva York: McGraw Hill. 
Jocumsen, G. (2004). How do small business managers make strategic marketing decisions? European Journal of Marketing 38 (5/6): 659-674.

Jordan, A. T. (2003). Business anthropology. Long Grove: Waveland Press Inc.

Fang, T. (2003). A critique on Hofstede's fifth national culture dimension. International Journal of Cross Cultural Management 3: 347-368.

Franke, R., G. Hofstede y M. Bond (1991). Cultural roots of economic performance: a research note. Strategic Management Journal 12: 165-173

Gibbons, P. T. y T. O'Connor (2005). Influences on strategic planning processes among Irish SMEs. Journal of Small Business Management 43 (2): 170186.

Liu, H. y L-U.Roos (2006). Managing strategic paradigms in China. Marketing intelligence and planning 24 (5): 432-445.

López, L. y G. Calderón (2006). Análisis de las dinámicas culturales al interior de un cluster empresarial. Estudios gerenciales 99: 13-37.

Masurel, E. y H. Smit (2000). Planning behavior in small firms in Central Vietnam. Journal of small business management 38 (2): 95-102.

McDonald, M., M.C. Spears y D.F. Parker (2004). Strategic management: does personality make a difference? Academy of strategic management Journal, vol. 3: 37-45.

Miles, R. E. y C.C.Snow (1978). Organization, strategy, structure, process. Nueva York: McGraw-Hill.

Mintzberg, H. (1981). What is planning anyway? Strategic Management Journal: 319-325.

Mintzberg, H. y J. Waters (1982). Tracking strategy in an entrepreneurial firm. Academy of Management Journal 25 (3): 465-499. 
Mulhare, E. (1999). Mindful of the future: strategic planning ideology and the culture of non profit management. Human Organization 58 (3): 323-330.

Payne, T., K. Kennedy, J.D. Blair y M.D. Fottler (2005). Strategic cognitive maps of small business leaders. Journal of Small Business Strategy 16 (1): 27-40.

Porter, M. (1990). Ventaja competitiva: creación y sostenimiento de un desempeño superior. México Diana.

Rauch, A., M. Frese. y S. Sonnentag (2000). Cultural differences in planning/success relationships: A comparison of small enterprises in Ireland, West Germany and East Germany. Journal of Small Business Management 38 (4): $28-41$.

Tower, C. B, D. Gudmundson, S. Schierstedt, y E.A. Hartman(2007). Do family meetings really matter? Their relationship to planning and performance outcomes in small family business. Journal of Small Business Strategy 18 (1): 85-93.

Trompenaars F. y C. Hampden-Turner (1998). Riding the waves of culture. Nueva York: Mc Graw Hill. 


\section{Anexo 1}

\section{Cuestionario internacional (vsm 94)}

Piense por favor en un trabajo ideal — sin tener en cuenta su trabajo actual, si lo tiene. Al escoger un trabajo ideal, qué grado de importancia tendría para usted... (marque, por favor, una casilla por línea).

\begin{tabular}{c|l|c|c|c|c|c}
\hline \multicolumn{1}{c|}{ Pregunta } & $\begin{array}{c}\text { De } \\
\text { muchísima } \\
\text { importancia }\end{array}$ & $\begin{array}{c}\text { Muy } \\
\text { importante }\end{array}$ & $\begin{array}{c}\text { De } \\
\text { moderada } \\
\text { importancia }\end{array}$ & $\begin{array}{c}\text { De poca } \\
\text { importancia }\end{array}$ & $\begin{array}{c}\text { De muy poca } \\
\text { o nada de } \\
\text { importancia }\end{array}$ \\
\hline $\mathbf{2}$ & $\begin{array}{l}\text { Tener suficiente } \\
\text { tiempo para su vida } \\
\text { personal o familiar }\end{array}$ & $\mathbf{1}$ & $\mathbf{2}$ & $\mathbf{3}$ & $\mathbf{4}$ & $\mathbf{5}$ \\
\hline $\begin{array}{l}\text { de trabajo (por ej.): } \\
\text { buena ventilación, } \\
\text { buena luz, espacio } \\
\text { de trabajo adecuado, } \\
\text { etc. }\end{array}$ & $\mathbf{1}$ & $\mathbf{2}$ & $\mathbf{3}$ & $\mathbf{4}$ & $\mathbf{5}$ \\
\hline $\mathbf{3}$ & $\begin{array}{l}\text { Tener una buena } \\
\text { relación con su } \\
\text { superior directo }\end{array}$ & $\mathbf{1}$ & $\mathbf{2}$ & $\mathbf{3}$ & $\mathbf{4}$ & $\mathbf{5}$ \\
\hline $\mathbf{4}$ & $\begin{array}{l}\text { Tener seguridad de } \\
\text { trabajo }\end{array}$ & $\mathbf{1}$ & $\mathbf{2}$ & $\mathbf{3}$ & $\mathbf{4}$ & $\mathbf{5}$ \\
\hline $\mathbf{5}$ & $\begin{array}{l}\text { Trabajar con } \\
\text { personas que } \\
\text { colaboran bien con } \\
\text { las otras }\end{array}$ & $\mathbf{1}$ & $\mathbf{2}$ & $\mathbf{3}$ & $\mathbf{4}$ & $\mathbf{5}$ \\
\hline $\mathbf{6}$ & $\begin{array}{l}\text { Ser consultado por } \\
\text { su superior directo } \\
\text { en sus decisiones } \\
\text { conseguir trabajos } \\
\text { de más alto nivel } \\
\text { trabajo }\end{array}$ & $\mathbf{1}$ & $\mathbf{2}$ & $\mathbf{3}$ & $\mathbf{4}$ & $\mathbf{5}$ \\
\hline & $\begin{array}{l}\text { Tener un elemento } \\
\text { Tener la }\end{array}$ & $\mathbf{1}$ & $\mathbf{3}$ & $\mathbf{5}$ & $\mathbf{5}$ \\
\hline
\end{tabular}


En su vida privada, ¿qué grado de importancia tiene para usted lo siguiente? (marque, por favor, una casilla por línea).

\begin{tabular}{c|l|c|c|c|c|c}
\hline \multicolumn{2}{c|}{ Pregunta } & $\begin{array}{c}\text { De } \\
\text { muchísima } \\
\text { importancia }\end{array}$ & $\begin{array}{c}\text { Muy } \\
\text { importante }\end{array}$ & $\begin{array}{c}\text { De } \\
\text { moderada } \\
\text { importancia }\end{array}$ & $\begin{array}{c}\text { De poca } \\
\text { importancia }\end{array}$ & $\begin{array}{c}\text { De muy poca } \\
\text { o nada de } \\
\text { importancia }\end{array}$ \\
\hline $\mathbf{9}$ & $\begin{array}{l}\text { Estabilidad } \\
\text { personal }\end{array}$ & $\mathbf{1}$ & $\mathbf{2}$ & $\mathbf{3}$ & $\mathbf{4}$ & $\mathbf{5}$ \\
\hline $\mathbf{1 0}$ & Economizar & $\mathbf{1}$ & $\mathbf{2}$ & $\mathbf{3}$ & $\mathbf{4}$ & $\mathbf{5}$ \\
\hline $\mathbf{1 1}$ & $\begin{array}{l}\text { Persistencia } \\
\text { (perseverancia) }\end{array}$ & $\mathbf{1}$ & $\mathbf{2}$ & $\mathbf{3}$ & $\mathbf{4}$ & $\mathbf{5}$ \\
\hline $\mathbf{1 2}$ & $\begin{array}{l}\text { Respeto a la } \\
\text { tradición }\end{array}$ & $\mathbf{1}$ & $\mathbf{2}$ & $\mathbf{3}$ & $\mathbf{4}$ & $\mathbf{5}$ \\
\hline
\end{tabular}

\begin{tabular}{|c|c|c|c|c|c|c|}
\hline \multicolumn{2}{|r|}{ Pregunta } & \multirow{2}{*}{$\begin{array}{c}\text { Nunca } \\
1 \\
1\end{array}$} & \multirow{2}{*}{$\begin{array}{c}\begin{array}{c}\text { Raras } \\
\text { veces }\end{array} \\
2\end{array}$} & \multirow{2}{*}{$\begin{array}{c}\begin{array}{c}\text { Algunas } \\
\text { veces }\end{array} \\
\text { 3 }\end{array}$} & \multirow{2}{*}{$\begin{array}{c}\text { A } \\
\text { menudo } \\
\\
4\end{array}$} & \multirow{2}{*}{$\begin{array}{c}\text { Siempre } \\
\mathbf{5}\end{array}$} \\
\hline 13 & $\begin{array}{l}\text { ¿Con qué frecuencia } \\
\text { se siente nervioso } \\
\text { o tenso en el trabajo? }\end{array}$ & & & & & \\
\hline
\end{tabular}

\begin{tabular}{c|c|c|c|c|c|c}
\hline \multicolumn{2}{c|}{ Pregunta } & $\begin{array}{c}\text { Casi } \\
\text { nunca }\end{array}$ & $\begin{array}{c}\text { Raras } \\
\text { veces }\end{array}$ & $\begin{array}{c}\text { Algunas } \\
\text { veces }\end{array}$ & Frecuentemente & $\begin{array}{c}\text { Muy } \\
\text { frecuentemente }\end{array}$ \\
\hline $\mathbf{1 4}$ & $\begin{array}{l}\text { ¿Con qué frecuencia, } \\
\text { según su experiencia, } \\
\text { sienten los } \\
\text { subordinados temor a } \\
\text { expresar su } \\
\text { disconformidad con sus } \\
\text { superiores? }\end{array}$ & $\mathbf{1}$ & $\mathbf{2}$ & $\mathbf{3}$ & $\mathbf{4}$ & $\mathbf{5}$ \\
\hline
\end{tabular}


¿En qué medida está de acuerdo o en desacuerdo con las siguientes afirmaciones?

\begin{tabular}{|c|c|c|c|c|c|c|}
\hline \multicolumn{2}{|r|}{ Pregunta } & \multirow{2}{*}{$\begin{array}{c}\text { Totalmente } \\
\text { de acuerdo }\end{array}$} & \multirow{2}{*}{$\begin{array}{l}\text { De } \\
\text { acuerdo } \\
2\end{array}$} & \multirow{2}{*}{$\begin{array}{c}\begin{array}{c}\text { Ni de } \\
\text { acuerdo } \\
\text { ni en } \\
\text { desacuerdo }\end{array} \\
\mathbf{3}\end{array}$} & \multirow{2}{*}{$\begin{array}{c}\begin{array}{c}\text { En } \\
\text { desacuerdo }\end{array} \\
4\end{array}$} & \multirow{2}{*}{$\begin{array}{c}\begin{array}{c}\text { Totalmente } \\
\text { en } \\
\text { desacuerdo }\end{array} \\
\mathbf{5}\end{array}$} \\
\hline 15 & $\begin{array}{l}\text { Se puede confiar en la } \\
\text { mayoría de las personas }\end{array}$ & & & & & \\
\hline 16 & $\begin{array}{l}\text { Se puede ser un buen } \\
\text { director sin tener respuestas } \\
\text { exactas a la mayoría de } \\
\text { las preguntas que los } \\
\text { subordinados puedan hacer } \\
\text { acerca de su trabajo (de los } \\
\text { subordinados) }\end{array}$ & 1 & 2 & 3 & 4 & 5 \\
\hline 17 & $\begin{array}{l}\text { Se debe evitar a todo } \\
\text { coste una estructura de } \\
\text { organización en la cual } \\
\text { ciertos subordinados } \\
\text { tienen dos jefes }\end{array}$ & 1 & 2 & 3 & 4 & 5 \\
\hline 18 & $\begin{array}{l}\text { La competencia entre } \\
\text { empleados normalmente } \\
\text { causa más daño que bien }\end{array}$ & 1 & 2 & 3 & 4 & 5 \\
\hline 19 & $\begin{array}{l}\text { Las reglas de una } \\
\text { organización o compañía } \\
\text { no se deberían infringir, } \\
\text { incluso cuando el empleado } \\
\text { piensa que es por el bien de } \\
\text { la compañía }\end{array}$ & 1 & 2 & 3 & 4 & 5 \\
\hline 20 & $\begin{array}{l}\text { Cuando la gente fracasa en } \\
\text { la vida, es a menudo por su } \\
\text { propia culpa }\end{array}$ & 1 & 2 & 3 & 4 & 5 \\
\hline
\end{tabular}

\section{Información personal}

\section{Usted es: 1. Hombre 2. Mujer}

\section{Su edad es:}

\begin{tabular}{l|l}
\hline 1. & Menos de 20 \\
\hline 2. & $20-24$ \\
\hline 3. & $25-29$ \\
\hline 4. & $30-34$ \\
\hline 5. & $35-39$ \\
\hline 6. & $40-49$ \\
\hline 7. & $50-59$ \\
\hline 8. & 60 o más \\
\hline
\end{tabular}


23. ¿Cuántos años de educación escolar formal (o su equivalente) ha realizado en total?

\begin{tabular}{l|l}
\hline 1. & 10 años o menos \\
\hline 2. & 11 años \\
\hline 3. & 12 años \\
\hline 4. & 13 años \\
\hline 5. & 14 años \\
\hline 6. & 15 años \\
\hline 7. & 16 años \\
\hline 8. & 17 años \\
\hline 9. & 18 años o más \\
\hline
\end{tabular}

24. Si tiene o ha tenido un trabajo remunerado, ¿de qué tipo de trabajo se trata?

\begin{tabular}{l|l}
\hline 1 & Trabajo no remunerado (incluso estudiantes) \\
\hline 2 & Trabajo manual que no requiere experticia o solo cierta experticia \\
\hline 3 & Trabajo general de oficina o secretaria \\
\hline 4 & $\begin{array}{l}\text { Artesano, técnico, informático, enfermera, artista o equivalente formado } \\
\text { proprofesionalmente }\end{array}$ \\
\hline 5 & Profesional formado académicamente o equivalente (pero no director) \\
\hline 7 & Director de uno o más subordinados (no-directores) \\
\hline
\end{tabular}

25. ¿Hace usted planeación estratégica? $\quad 1$. Sí 2. No

26. ¿Cuál es su nacionalidad?

27. ¿Cuál es su nacionalidad de nacimiento?

28. Nombre la empresa:

29. Nombre encuestado:

30. Dirección empresa:

31. e-mail:

32. Teléfono:

33. Nombre del encuestador: 


\section{Anexo 2}

\section{Listado de empresas participantes en fase cuantitativa}

PEQUEÑAS EMPRESAS: 1) CRC Outsorcing S.A; 2) Productos Joyce; 3) Ger'art; 4) Piqueteadero «Doña Nieves»; 5) Univertel; 6) Dar papaya; 7) Pandora postres; 8) Tidbit; 9) Vicpar; 10) PCPOWER; 11) Doble A ingeniería S.A; 12) ADGORA; 13) SITIO DE CONTACTO; 14) VIAJES GALEON; 15) Reuters Ltda.; 16) VINACURE; 17) Store Tattoo; 18) Idustrias Label; 19) Acid Ink; 20) Brian Frutería Panadería; 21) Yoyo music; 22) Cinemateca; 23) Inmobiliaria Andes de Colombia; 24) COLPARTEC; 25) QUIDECA S.A; 26) TELEHIT; 27) Sintonizar Medios Ltda.; 28) UNISISTEMS; 29) WOOD SOLUTIONS; 30) PSO COLOMBIA; 31) SERTIC; 32) DANNY VENTA DIRECTA; 33) DANIEL SUAREZ ARQUITECTOS; 34) FANARCHIVOS; 35) INBOX DISEÑO LTDA; 36) Óptica Visico Colombia; 37) JAIME BENITEZ; 38) Mundo Fauna Pet Shop; 38) Mascotas y mucho más; 39) Manos de Colombia; 40) Aquí en Santa Fe; 41) Serproint; 42) Comercial Papelera; 43) San Marcos; 44) Patacones Food and Galery; 45) Canal Clima; 46) La Biferia; 47) H Y B BAR; 48) Restaurante ZHANG; 49) Dilleto café; 50) Bibliotecnica; 51) Diafana; 52) Kaldivia Marly; 53) Disalud: Ortopédicos y droguería; 54) Stuff accesorios; 55) Estudio de Grabación Audio Ocho; 56) Parix Wash; 57) AG impresores; 58) AUROS COPIAS; 59) Plastifer Ltda.; 60) Tenidata de Colombia; 61) Touret; 62) Gontec Ltda.; 63) Sercar Ltda.; 64) Coltapetes; 65) Cyclus; 66) All service tecnology; 67) Industria deportiva Volcan; 68) Fer-Arte y Flores; 69) Detectives privados profesionales; 70) D\&D Accesorios; 71) CONTADORES AUDITORES FISCALES; 72) EVENPRO; 73$) 57$ UNO ESTUDIO; 74) FINLECO; 75) FEEL; 76) Almacolor Ltd.; 77) Mediservice; 78) Pan Fino; 79) Espejo Azul; 80) Home Medical; 81) LA RESPUESTA; 82) EDWIM RAMOS PELUQUERÍA; 83) INTERCAMBIOS CULTURALES; 84) Gran Pared Ltda.; 85) Scale Ploter; 86) Edwin Ramos Peluquería; 87) Bogotana De Belleza; 88) FW Fashion World; 89) KONKER; 90) IND.COCINAS FRANCIA; 91) GAITAN PARRA \& GAVIRIA Ltda.; 92) The Dot Studio; 93) Inversiones El Bambú; 94) Bermotos; 95) Choco Choice; 96) Joyería el Brillante; 97) Optiproductos; 98) Movilicentro Ltda.; 99) PARK WASH; 100) JUAN MARIANO NARANJO - SCALE PLOTTER; 101) MUFFINS \& CREPES; 102) Colombian PUB; 103) Manos a la Obra; 104) San Valentín; 105) Papeles RR Ltda.; 106) Borrego Ltda.; 107) Gráfica Davinson; 108) CEVICHERIA TAGANGA; 109) CARRUSEL JARDIN INFANTIL; 110) GALERIA HERMIDA; 111) CENTRO DE ANALISIS AL CONSUMIDOR; 112) SPINNING CENTER; 113) Químicos 
Campota y Cía Ltda.; 114) Total Ltda.; 115) Big Class Peluquería; 116) BKF Fernández International; 117) Borrero Ochoa y Asociados Ltda.; 118) Apartahotel 51; 119) Tiyuca Ltda.; 120) K9 Security Ltda.; 121) Avaya; 122) FRANQUICIA CENTRO; 123) BOSTON MEDICAL DEVICE; 124) CONFORT Y ASOCIADOS; 125) FRANQUICIA NORTE. COLP; 126) NBC SEGUROS Y CAPITALIZACION; 127) Cambios Chapinero; 128) Lava-rápido Chapinero alto; 129) Planpizza; 130) ABG S\&S; 131) COORATIENDAS; 132) SPRACH INSTITUT; 133) LA AUTÉNTICA HAMBURGESA; 134) PELUQUERÍA SENSACIONES; 135) SLIDE DEPOT; 136) FÁBRICA COLOMBIANA DE SELLOS; 137) PHOTO IMAGES LTDA.; 138) JEMMAUS CARGO LOGISTICS; 139) CABARRIA \& CÍA.; 140) Oficomputo Ltda.; 141) Flores en tu casa Ltda.; 141) Easy Solutions; 142) Centro de Alta Tecnología; 143) Makrovision Ltda.; 144) DENTALES PADILLA; 145) SERVIOPEN LTDA.; 146) POSICIONATE LTDA.; 147) TEAM 4 DIGITAL STUDIO; 148) SISTEMAS DMS LTDA.; 149) MACELTEX \& CIA; 150) Monici Ltda.; 151) Gráficas Bacatá; 152) Creatisoft Ltda.; 153) SIA security and Intelligence advising Ltda.; 154) Diseño y moda desing school Ltda.; 155) ELECTRIMEC; 156) LHAURAVET; 157) FOTO LASER; 158) DATA CONTROL; 159) TIBER LTDA.; 160) IGLESIA CRISTIANA FILADELFIA; 161) TEATRO LIBRE; 162) Interlux Ltda.; 163) Ensistema; 164) Mauris Copias; 165) Peña y Asociados; 166) United Motors; 167) Fatelares; 168) El Patriarca; 169) Animal center; 170) Inversiones Sim; 171) Central Parking; 172) Parking solutions Colombia; 173) Rafael DC; 174) PROMOTORA NUEVA ANDALUCIA DEL MAR; 175) WALL STREET; 176) AUTO ELITE; 177) INVERSIONES VARGHI; 178) Segno musical; 179) Híbrido; 180) Fabian hb peluquería; 181) Animal Center; 182) Don Eloy Rosas; 183) Espacio Ilimitado; 184) Sellos y Banca Ltda.; 185) MSCrea; 186) Papelería La 53; 187) Sala de Belleza Nuby; 188) Ferretería Zachariel; 189) Cigarrería Jotas; 190) Lavado Express; 191) Plotter Net; 192) Alpenhaus; 193) Perfes; 194) Gran Pared; 195) El punto dulce; 196) La Ropería; 197) Maroma; 198) Philomena; 199) Cachao; 200) Gavanna; 201) Beer Lounge; 202) Aseo el Esplendor; 203) Solarko Ltda.; 204) Biocontrol Ltda.; 205) Noctapolis; 206) Blossom Bar; 207) Puebla Restaurante; 208) Planeta Cao; 209) Invitro Bar; 210) Novakem S.A.; 211) Bellisima; Bickenvach y Vieites S.A.; 212) Tramec Ltda.; 213) Publicis Colombia S.A.; 214) Vedi Travel; 215) Bustamante Deportes Ltda.; 216) Arte Clau; 217) Mendebal; 218) Affinity Network S.A.; 219) KTL; 220) Shalom; 221) Barbe Club; 222) Digitiva Group; 223) Grupo inventa; 224) Oscar market; 225) C.G,G; 226) Traducciones Praxis; 227) My Home; 228) Academia Liverpool; 229) Starcom; 230) LGL digital media; 231) 1492 bar; 232) Sphera; 233) Editorial y Librería Herder Ltda.; 234) Alianza Distribuidora; 235) 
Círculo de Lectores; 236) Abastanza; 237) Publicis; 238) Metis; 239) Beer Lounge; 240) JAS Forwarding Colombia; 241) Abbot; 242) PWH; 243) Multisoft; 244) La Boca del Lobo; 245) Casa Don Juaco; 246) Dental Art; 247) Credi flores; 248) Notaria 27; 249) Dentis Plus; 250) Janeth montero Estética y peluquería; 251) Talent; 252) Muelle Mackencie; 253) DENTALES PADILLA LTDA.; 254) SUS ASESORES UNO A; 255) EL CLAM S.A.; 256) XOREX; 257) SCOPE; 258) EL SITIO GROUP S.A.; 259) MARKETING IMAGEN Y PUBLICIDAD; 260) 141 COLOMBIA; 261) PROTECCION Láminas y películas; 262) MUSSINI; 263) IN4; 264) Outsourcing JN Ltda; 265) Solucionescolombia; 266) Sistemas DMS Ltda.; 267) SIA Security and Intelligence Advising Ltda.; 268) Sena Tecnoparque; 269) Bicultural Ltda.; 270) LASSER LTDA; 271) Fiduciaria Bogotá; 272) Business Parner consulting; 273) Aguazul; 274) Construarq Ltda.; 275) Gomez Cajiao \& Asociados S. A.; 276) El punto dulce; 277) Alpenhaus; 278) Mi sabana Café bar; 279) Perfes; EMPRESAS MEDIANAS: 280) BRISA S.A.; 281) CORALTEX LAVADO EN SECO; 282) PLAYHAIRCOLORS; 283) VAYCLEAN; 284) DROGUERÍA SUPER ANDINA; 285) QUESOS Y QUESOS; 286) SOLUCIONES LASER; 287) PANADERIA PASTELERIA MARLY; 288) BILLMES LTDA; 289) PELUQUERÍA AUGUSTO MORALES; 290) DOÑA PIZZA PUNTO Y COMA; 291) RED MOSQUITO; 292) ALICO S.A.; 293) Efe X; 294) Logograma; 295) Ingeniería y mantenimiento S.A.; 296) Patria S.A.; 297) Murcia - Murcia S.A.; 298 )C.I Carbocoque S.A.; 299) M+ Ltda.; 300) Restaurante El Portal de la Antigua; 301) Color Piel; 302) Sala de belleza Luis XV; 303) Bazar Persa; 304) Surtiaves 22; 305) Mis carnes y sopas Bayacences; 306) Muebles casa estilo; 307) Bassler; 308) Politecnico Internacional Sonría; 309) Concesionario RA; 310) Camisetas Col.; 311) TopWear; 312) Momfood's; 313) Look style; 314) Pastelería Holandesa; 315) Café con algo rico; 316) TREETOP ROCKET S.A.; 317) COMPUNET S.A.; 318) ALFAPARF; 319) COENPLAS; 320) LAB. SYNTHESIS; 321) Digueli Ltda; 322) Sopitas y Frijoladas Restaurante; 323) Lincoln English Center; 324) Pizza FX; 325) Copyuniversitaría; 326) Droguería 47; 327) Don Pepe; 328) Uganda Cafetería; 329) Net \& Cía. 
